\title{
Diagnosis and management of sepsis
}

\author{
Author: Tom Evans ${ }^{\mathrm{A}}$
}

Sepsis is a common condition with high morbidity and mortality. Although many patients may require critical care, this article considers the features of sepsis that are of most relevance to acute general physicians. Recently updated definitions of sepsis and septic shock have been proposed which better identify patients who are likely to have a poor outcome, and therefore give an opportunity to escalate care. Despite these advances, there is still no molecular signature able to diagnose sepsis. Pathogenesis is complex, with many immune and non-immune mediators involved. Four key areas are endothelial dysfunction, coagulation abnormalities, alterations in cell function and dysregulated cardiovascular responses. No specific therapy targeting the mediators of sepsis has yet proven effective. Prompt administration of appropriate antibiotics is of benefit, together with fluid resuscitation and oxygen.

\section{Introduction}

Sepsis derives from the Greek for putrefaction and was recognised as a frequent complication of wounds. As will be discussed here, the definitions of sepsis still prove difficult, and despite significant advances in intensive care medicine and antimicrobial therapy, sepsis remains a common condition with high attributable mortality. The most seriously affected by sepsis will require management in an intensive care setting where available, but this article will focus on the diagnosis and immediate management of sepsis, as this is of broad importance to all physicians dealing with acutely ill patients.

\section{Definition}

Septicaemia, blood poisoning and other vague terminology, meaning different things to different people, were used frequently. This hampered attempts to properly identify septic patients promptly, and hindered the design of trials with standardised entry criteria. In 1991, a consensus conference developed definitions of sepsis that linked infection with a systemic inflammatory response, the systemic inflammatory response syndrome (SIRS), which was defined using simple clinical and laboratory parameters. ${ }^{1,2}$ This had the advantage of standardising the definition of sepsis across

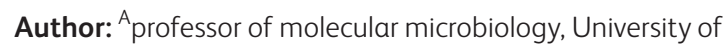
Glasgow, Glasgow, UK different regions and was a significant advance. However, the definition of SIRS, although sensitive to detect sepsis, was rather unspecific, since similar inflammatory responses were seen as part of the physiological response to non-infectious insults, such as surgery and pancreatitis. In addition, the SIRS criteria performed badly in identifying patients who required critical care and who had significant morbidity and mortality. ${ }^{3}$

These issues led to a recent new consensus definition for sepsis and septic shock. ${ }^{4-6}$ This international task force defined sepsis as 'life-threatening organ dysfunction caused by a dysregulated host response to infection.' Importantly, the task force considered how to operationalise this definition, by analysing what clinical criteria best identified infected patients with sepsis who died. Using large datasets ( $>1$ million patient records), they found that an increase in 2 points or more for a patient suspected to have infection using the Sequential Organ Failure Assessment (SOFA) best predicted in-hospital mortality. ${ }^{6}$ The SOFA is well known within the intensive care community, but is not so well known generally. Therefore, the task force developed a simpler clinical screening tool that performed very well in identifying adult patients with suspected infection who were likely to have poor outcomes, which they termed 'quick SOFA' (qSOFA). This measures three clinical parameters (Box 1).

A patient who fulfilled two of these criteria had similar outcomes to those who had an increase in 2 points on the full SOFA scale. The task force therefore considered that a qSOFA score of 2 or

\section{Key points}

A new definition of sepsis allows identification using a quick SOFA score (qSOFA)

Septic shock can be recognised by the combination of hypotension and increased blood lactate $>2 \mathrm{mM}$

Prompt administration of empirical antibiotics is essential in suspected sepsis

Use of the 'Sepsis six' bundle will ensure immediate management of patients with sepsis is optimal

Early recognition of sepsis is crucial to better management

KEYWORDS: sepsis, infection, critical care 
Box 1. qSOFA criteria
> Respiratory rate $\geq 22 / \mathrm{min}$
$>$ Glasgow Coma Scale $<15$
Systolic blood pressure $\leq 100 \mathrm{mmHg}$

more should prompt clinicians to investigate further for organ dysfunction, consider escalation of therapy, and evaluate for referral to critical care.

The same grouping also considered the definitions of septic shock. Traditionally, this had been defined based on the cardinal feature of hypotension as a marker of the cardiovascular dysfunction associated with this condition. However, it has become apparent that the application and interpretation of such a definition is very variable. Therefore, a new consensus was sought. Septic shock was newly defined as 'a subset of sepsis in which circulatory, cellular and metabolic abnormalities are associated with a greater risk of mortality than sepsis alone. ${ }^{5}$ Operationally, they identified septic shock as being present when the two particular conditions were met (Box 2).

These new definitions have been adopted by numerous international societies but are only just entering into routine clinical practice. They have the advantage of simplicity and are underpinned by robust data on their performance in identifying those patients with suspected infection who have the worst outcomes. They are simpler to apply, for example, than the recent National Institute for Health and Care Excellence (NICE) guidelines. ${ }^{7}$ However, a number of problems remain. This consensus group did not address the definition of infection. Despite improvements in diagnosis, only about $30-40 \%$ of patients who are suspected of having an infection will actually have a positive microbiological diagnosis. ${ }^{8}$ Therefore, patients whose organ dysfunction is secondary to a cause other than infection will still potentially be misdiagnosed and unnecessary antimicrobials administered. Additionally, no stratification of different degrees of sepsis is given, so targeting care for individual patients is difficult. In other fields, such as cancer biology, a wealth of genetic, transcriptomic and metabolic data are allowing ever more specific 'personalised' treatment. Despite many excellent studies, there is as yet no molecular 'signature' of particular biomarkers that can unequivocally identify a patient with infection or their likely outcome. Such objective molecular criteria would greatly help in diagnosis and management.

\section{Pathogenesis}

The pathogenesis of sepsis is complex ${ }^{9,10}$ and due to the constraints of space, only a summary can be given here. A key conceptual advance was made in a seminal paper in 1986 which showed that the host cytokine tumour necrosis factor (TNF, also known as cachectin) could reproduce many of the pathological and clinical features of sepsis. ${ }^{11}$ Given that many bacterial and

\section{Box 2. Criteria for septic shock}

$>$ A need for vasopressor therapy to maintain a mean arterial pressure $\geq 65 \mathrm{mmHg}$

$>$ A serum lactate $>2 \mathrm{mM}$, persisting after adequate fluid resuscitation other pathogen products can induce TNF production, it was clear that the host response to infection played an important role in the pathogenesis of the condition, as originally observed by Lewis Thomas in 1972. Since then, numerous studies have shown a complex network of cytokines are important in mediating many of the effects of sepsis. Moreover, although initial proinflammatory pathways are important, anti-inflammatory pathways are also activated and can downregulate corrective responses later in the course of sepsis. In addition to protein and peptide mediators, there are also a plethora of other mediators involved, including prostanoids, platelet activating factor, and endogenous damage-associated molecular patterns (DAMPS) released from injured cells, such as ATP and high mobility group proteins.

In an attempt to simplify this complex pathogenic pathway, four main features can be highlighted - the four horsemen of the septic apocalypse (Fig 1).

\section{$>$ Endothelial dysfunction}

Generalised endothelial activation increases the expression of a number of leucocyte adhesins, with increased leucocyte transmigration into tissues. The permeability of the endothelium is also increased, in the lung leading to interstitial pulmonary edema and in the gut increasing bacterial translocation, potentially exacerbating the inflammatory cascades already initiated by microbial products.

\section{$>$ Coagulopathy}

Altered coagulation is extremely common in sepsis. Endothelial damage removes the protective function of the natural anticoagulation protein C pathway and converts the endothelium into a prothrombotic surface. In addition, bacterial products and inflammatory cytokines activate tissue factor, the main initiator of the extrinsic pathway of blood coagulation. This prothrombotic state may lead to blockage of the microvasculature, as well as giving rise to a consumption coagulopathy (disseminated intravascular coagulation). Gram-positive products can also directly activate the contact clotting system.

\section{$>$ Cellular dysfunction}

One of the enigmas of the field is that even in the most severe cases of lethal sepsis, autopsy studies show little evidence of cell death, despite widespread organ dysfunction. The molecular basis of this is still not clear, although a generalised reduction in energy expenditure by cells suggests some kind of hibernationlike process. Concomitant with this alteration in cellular function are numerous metabolic changes, notably increased catabolism, insulin resistance and hyperglycaemia.

$>$ Cardiovascular dysfunction

Many studies have shown that patients with sepsis have a decreased systemic vascular resistance (SVR) with a normal or increased cardiac output, the so-called 'hyperdynamic' state of sepsis. Cardiac output is maintained at the expense of left ventricular dilation, with reduced ejection fraction and a reduced left ventricular stroke work index in response to left ventricular end diastolic volume increase. These changes can lead to the hypotension characterising septic shock. Changes in SVR are probably largely mediated by excess production of the vasodilator nitric oxide in the vasculature, ${ }^{12}$ which can be difficult to correct with vasopressors. Poor tissue perfusion also likely underlies the increased lactate seen in septic shock, although other mechanisms are possible. 
Fig 1. The key pathophysiological changes of sepsis and how these combine to produce multiorgan failure.

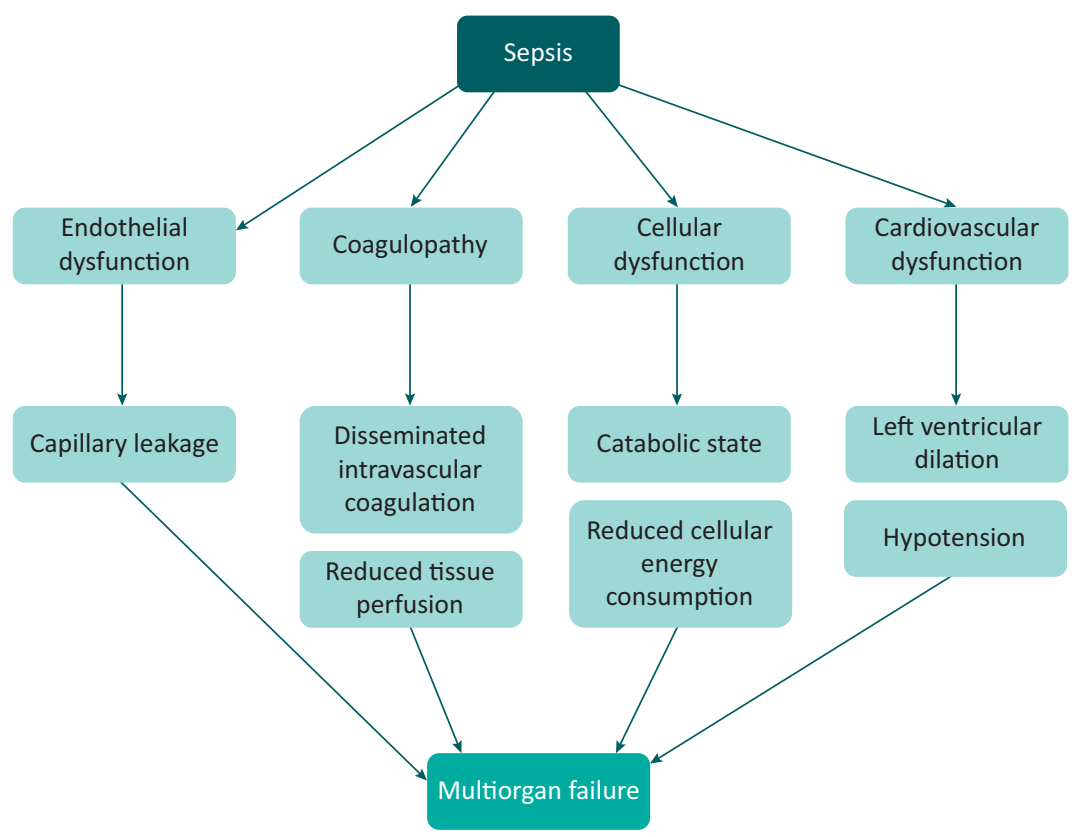

\section{Management}

Survival in sepsis has improved over the last 40 years. However, we still lack a specific molecular therapy for this condition, other than antimicrobial therapy. Numerous trials of promising biological agents targeting different mediators of sepsis have failed. This article will focus on the immediate management of sepsis - the management of patients in a critical care setting is not covered here.

\section{$>$ Resuscitation}

Immediate resuscitation of a critically ill septic patient is not appreciably different from non-septic patients. Adequate oxygen to maintain saturations in excess of $95 \%$ should be given. Although there is no high quality randomised controlled trial evidence, it is considered standard care to give intravenous saline to all patients with sepsis. ${ }^{13}$ For patients with hypotension, this should be a bolus of $500 \mathrm{~mL}$ of saline over 15 minutes. Further fluids should be titrated to response. Starch based fluids should be avoided $^{14}$ and there is no evidence to support the use of albumin. ${ }^{15}$ Persistent hypotension despite adequate fluid resuscitation will almost certainly require admission to a critical care facility and the use of vasopressors - noradrenaline is the preferred agent. ${ }^{16}$

> Prompt and appropriate antimicrobial therapy

Studies have shown a clear benefit of rapid use of antimicrobials that target the likely causative pathogens. ${ }^{17}$ Although the exact timing required is not entirely clear, every effort should be made to give such drugs as quickly as possible, ideally within 1 hour of admission. Prior to administering antibiotics, blood cultures should be taken. Although there are no trials showing the benefit or not of such cultures, identification and characterisation of

\section{Table 1. Summary of different guidelines for the management of sepsis}

Origin

Sepsis Trust

National Institute for Health and Care Excellence (NICE) guidelines

Royal College of Physicians

Royal College of Emergency Medicine

Surviving sepsis campaign

\section{Website}

https://sepsistrust.org/education/clinical-tools/

www.nice.org.uk/guidance/ng51

www.rcplondon.ac.uk/guidelines-policy/acutecare-toolkit-9-sepsis

www.rcem.ac.uk/docs/Clinical\%20Standards\%20 and\%20Guidance/Clinical\%20Standards $\% 20$ for\%20Emergency\%20Departments.pdf www.survivingsepsis.org/Bundles/Pages/ default.aspx

\section{Focus}

UK-based charity and originator of the 'Sepsis six'

Produced in collaboration with NICE

Comprehensive though complex algorithms Published before new sepsis definitions

Distillation of NICE guidance and again uses older definitions

Based on Sepsis Trust guidelines Tailored to emergency department use

The most up to date and useful of the guidelines, using the new (2016) definitions of sepsis 
antibiotic sensitivities of cultured pathogens is crucial in further management.

$>$ Accurate fluid balance

Urine output should be recorded, together with all fluids administered. A urinary catheter should be placed if required for patient management but it is not essential.

$>$ Blood glucose

In the event of hyperglycaemia, blood sugar should be kept $<10 \mathrm{mM}$ with intravenous insulin. More aggressive blood sugar control is contraindicated. ${ }^{18}$

$>$ Source control

Notwithstanding the need for immediate attention to the deranged physiological parameters, identification and management of the source of sepsis is also important. From the history, full examination and appropriate radiological investigations, a likely source of infection may be identified; although, in around $25 \%$ of cases no source can be identified. However, prompt management of an infection source is vital, such as drainage of a pleural effusion, debridement of an infected wound, or surgical intervention to drain an intra-abdominal abscess.

A variety of care bundles to streamline the delivery of such care have been evaluated, see Table 1. In the UK, the 'Sepsis six' has been developed and adopted by the Sepsis Trust. ${ }^{19}$ It is a useful way of ensuring key steps in management are not forgotten and is widely used in UK hospitals. The most up-to-date guidelines are those published by the Surviving Sepsis Campaign. ${ }^{20}$

Despite an initial trial suggesting that early goal-directed therapy could reduce the mortality from septic shock, recent trials have not supported any benefit from such goal-directed therapy compared to standard care. ${ }^{21}$

\section{References}

1 Bone R, Fisher C], Clemmer TP et al. Sepsis syndrome: a valid clinical entity. Crit Care Med 1989;17:389-93.

2 Bone RC, Balk RA, Cerra FB et al. Definitions for sepsis and organ failure and guidelines for the use of innovative therapies in sepsis. The ACCP/SCCM Consensus Conference Committee. American College of Chest Physicians/Society of Critical Care Medicine. Chest 1992;101:1644-55.

3 Kaukonen KM, Bailey M, Pilcher D et al. Systemic inflammatory response syndrome criteria in defining severe sepsis. N Engl ] Med 2015;372:1629-38.

4 Singer M, Deutschman CS, Seymour CW et al. The Third International Consensus Definitions for Sepsis and Septic Shock (Sepsis-3). JAMA 2016;315:801-10.
5 Shankar-Hari M, Phillips GS, Levy ML et al. Developing a New Definition and Assessing New Clinical Criteria for Septic Shock: For the Third International Consensus Definitions for Sepsis and Septic Shock (Sepsis-3). JAMA 2016;315:775-87.

6 Seymour CW, Liu VX, Iwashyna T] et al. Assessment of Clinical Criteria for Sepsis: For the Third International Consensus Definitions for Sepsis and Septic Shock (Sepsis-3). JAMA 2016;315:762-74.

7 National Institute for Health and Care Excellence. Sepsis: recognition, diagnosis and early managment. London: NICE, 2016.

8 Cohen J, Vincent JL, Adhikari NK et al. Sepsis: a roadmap for future research. Lancet Infect Dis 2015;15:581-614.

9 Gotts JE, Matthay MA. Sepsis: pathophysiology and clinical management. BMJ 2016;353:i1585.

10 Cohen J. The immunopathogenesis of sepsis. Nature 2002:420:885-91.

11 Tracey KJ, Beutler B, Lowry SF et al. Shock and tissue injury induced by recombinant human cachectin. Science 1986;234:470-4.

12 Cobb JP, Danner RL. Nitric oxide and septic shock. J Amer Med Assoc 1996:275:1192-6.

13 Howell MD, Davis AM. Management of Sepsis and Septic Shock. JAMA 2017;317:847-8.

14 Perner A, Haase N, Guttormsen AB et al. Hydroxyethyl starch 130/0.42 versus Ringer's acetate in severe sepsis. N Engl ] Med 2012:367:124-34.

15 Caironi P, Tognoni G, Masson S et al. Albumin replacement in patients with severe sepsis or septic shock. N Engl J Med 2014:370:1412-21.

16 Levy MM, Artigas A, Phillips GS et al. Outcomes of the Surviving Sepsis Campaign in intensive care units in the USA and Europe: $a$ prospective cohort study. Lancet Infect Dis 2012;12:919-24.

17 Kumar A, Roberts D, Wood KE et al. Duration of hypotension before initiation of effective antimicrobial therapy is the critical determinant of survival in human septic shock. Crit Care Med 2006:34:1589-96.

18 Study Investigators NICE-SUGAR, Finfer S, Chittock DR et al. Intensive versus conventional glucose control in critically ill patients. N Engl J Med 2009:360:1283-97.

19 Daniels R, Nutbeam T, McNamara G, Galvin C. The sepsis six and the severe sepsis resuscitation bundle: a prospective observational cohort study. Emerg Med J 2011;28:507-12.

20 Rhodes A, Evans LE, Alhazzani W et al. Surviving Sepsis Campaign: International Guidelines for Management of Sepsis and Septic Shock: 2016 Crit Care Med 2017;45:486-552.

21 Investigators PRISM, Rowan KM, Angus DC et al. Early, goaldirected therapy for septic shock - A patient-level meta-analysis. N Engl J Med 2017;376:2223-34.

Address for correspondence: Professor Thomas Evans, Level 4, Glasgow Biomedical Research Centre, 120 University Avenue, Glasgow G12 8TA, UK.

Email: tom.evans@glasgow.ac.uk 